\section{Michelin Tyre Baby: A Rare Genodermatosis}

A 8-month-old female baby presented with cushingoid facies, with large skin folds involving the extremities and trunk since birth (Fig. 1). The child was born to healthy parents in a non-consanguineous marriage. The child weighed $8 \mathrm{kgs}$, was exclusively breast fed and developmentally normal and had no dysmorhic features. There was no history of similar complaints in other family members. Serum cortisol levels were normal. No abnormality was detected on abdominopelvic sonography.Skin biopsy revealed normal histology.

Michelin tyre baby syndrome is a rare benign genodermatosis resulting in multiple, symmetric skin folds since birth. This was first described by Ross in 1969, deriving its name from resemblance of the skin folds to the mascot of a French tire company. The skin folds are predominantly present on the extremities and trunk rarely on the palms and soles. Multiple congenital anomalies have been associated including low set ears, congenital heart defects, cleft palate and undescended testis. The syndrome may be familial. Skin biopsy may be normal. However there are biopsy reports suggesting diffuse lipomatous nevus in underlying dermis or smooth muscle hamartoma. Scarring instead of increased adipocytes have also been reported. The condition is benign and the skin folds gradually disappear. Differential diagnosis includes congenital amniotic

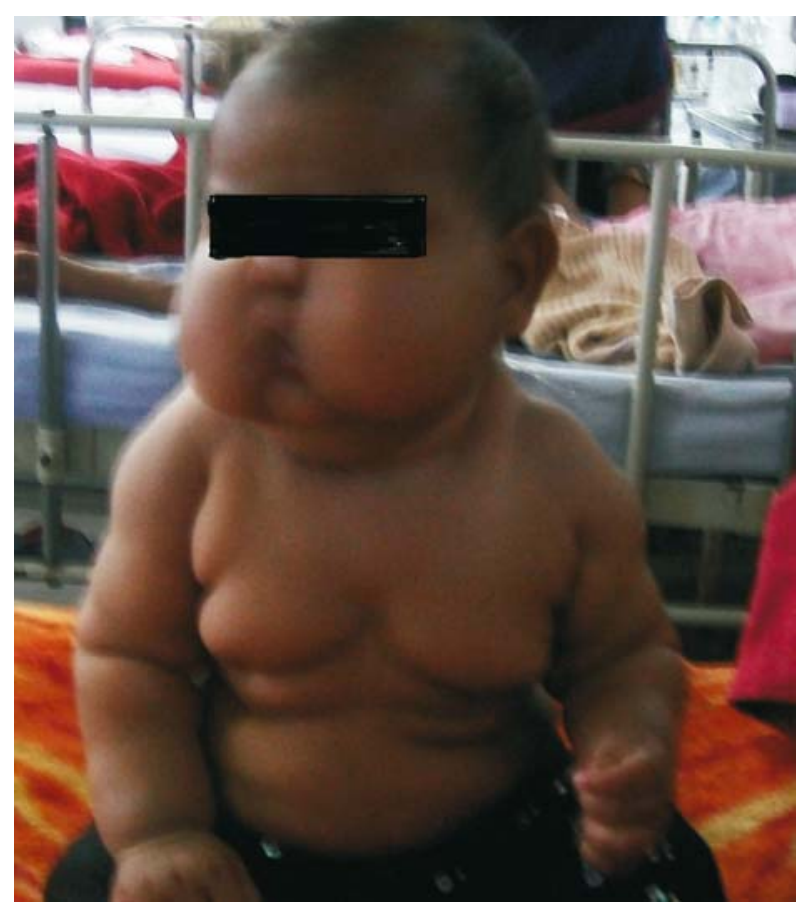

FIG. 1 Michelin tyre baby with skin folds over trunk.

bands; however these are usually solitary and limited to limbs. Beare Stevensons cutis gyrate syndrome is another syndrome characterized by dermatomegaly which is limited to scalp, forehead, face and neck.

Dhulika Dhingra, GR Sethi And Mukta Mantan Department of Pediatrics, Maulana Azad Medical College, New Delhi 110002 , India. drdhulika@yahoo.co.in

\section{Subungual Exostosis}

A 13-year-old boy presented with a 'growth' beneath the nail of the right great toe (Fig. 1). The lesion was painful and had been present for the preceding 6 months. The nodule was tender, bony-hard in consistency, and measured $20 \times 15 \mathrm{~mm}$ in diameter. The nail plate showed onycholysis. A radiograph revealed a calcified projection on the dorso-lateral part of the distal phalanx, continuous with the underlying bone (Fig. 2). Based on the clinical presentation and radiological features, a diagnosis of subungual exostosis was made.

Subungual exostosis is a relatively rare, acquired, benign osteocartilaginous tumor occurring mainly in children and young adults. They are found beneath the

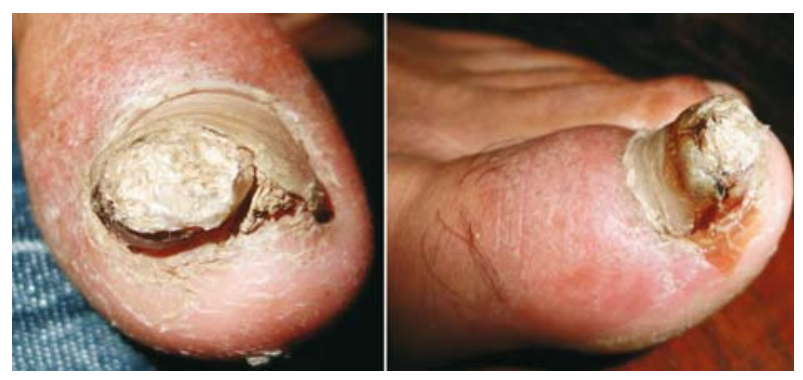

FIG. 1 Subungual nodule with onycholysis.

distal edge of the nail, most commonly of the great toe. However, other toes or, occasionally, a finger may be involved. The first manifestation of this tumor is a painful, small, pink or flesh-colored, hard, exophytic growth that projects beyond the inner free edge of the nail. The overlying nail becomes brittle and may be lifted 